\title{
ARTICLE
}

\section{Raman spectroscopy as probe of nanometre-scale strain variations in graphene}

\author{
C. Neumann ${ }^{1,2}$, S. Reichardt ${ }^{1}$, P. Venezuela ${ }^{3}$, M. Drögeler ${ }^{1}$, L. Banszerus ${ }^{1}$, M. Schmitz ${ }^{1}$, K. Watanabe ${ }^{4}$, \\ T. Taniguchi ${ }^{4}$, F. Mauri ${ }^{5}$, B. Beschoten ${ }^{1}$, S.V. Rotkin ${ }^{1,6}$ \& C. Stampfer ${ }^{1,2}$
}

Confocal Raman spectroscopy has emerged as a major, versatile workhorse for the non-invasive characterization of graphene. Although it is successfully used to determine the number of layers, the quality of edges, and the effects of strain, doping and disorder, the nature of the experimentally observed broadening of the most prominent Raman 2D line has remained unclear. Here we show that the observed 2D line width contains valuable information on strain variations in graphene on length scales far below the laser spot size, that is, on the nanometre-scale. This finding is highly relevant as it has been shown recently that such nanometre-scaled strain variations limit the carrier mobility in high-quality graphene devices. Consequently, the 2D line width is a good and easily accessible quantity for classifying the crystalline quality, nanometre-scale flatness as well as local electronic properties of graphene, all important for future scientific and industrial applications.

\footnotetext{
${ }^{1}$ JARA-FIT and 2nd Institute of Physics, RWTH Aachen University, Aachen 52074, Germany. ${ }^{2}$ Peter Grünberg Institute (PGI-9), Forschungszentrum Jülich, Jülich 52425, Germany. ${ }^{3}$ Instituto de Fsica, Universidade Federal Fluminense, Niterói, 24210-346 Rio de Janeiro, Brazil. ${ }^{4}$ National Institute for Materials Science,1-1 Namiki, Tsukuba 305-0044, Japan. ${ }^{5}$ IMPMC, UMR CNRS 7590, Sorbonne Universités-UPMC Univ. Paris 06, MNHN, IRD, 4 Place Jussieu, Paris 75005, France. ${ }^{6}$ Department of Physics and Center for Advanced Materials and Nanotechnology, Lehigh University, Bethlehem, Pennsylvania 18015, USA. Correspondence and requests for materials should be addressed to C.S. (email: stampfer@physik.rwth-aachen.de).
} 
G raphene combines several highly interesting material properties in a unique way, promising unprecedented material functionality. This makes graphene increasingly attractive for fundamental research as well as industrial applications ${ }^{1}$, but, at the same time, stresses the need for noninvasive characterization techniques. In recent years, Raman spectroscopy has proven to be highly useful as a non-invasive method not only to identify graphene ${ }^{2,3}$ but also to extract information on doping ${ }^{4-7}$, strain $^{8,9}$ and lattice temperature ${ }^{10,11}$. Even more insights can be gained when utilizing confocal, scanning Raman spectroscopy to study spatially resolved doping domains $^{7,12}$, edge effects 3,13 and position-dependent mechanical lattice deformations, including strain ${ }^{14-16}$. The spatial resolution of so-called Raman maps is on the order of the laser spot size (which for confocal systems is typically on the order of $500 \mathrm{~nm}$ ) and the extracted quantities (such as doping or strain) are in general averaged over the spot size. It is therefore important to distinguish between length scales significantly larger or smaller than the laser spot size. In particular, we will distinguish between strain variations on a micrometre scale, which can be extracted from spatially resolved Raman maps, and nanometre-scale strain variations, which are on sub-spot-size length scales and cannot be directly observed. Especially, nanometre-scale strain variations have been recently identified as the most important limitation to carrier mobility in high-quality graphene ${ }^{17}$, making this quantity increasingly important ${ }^{18}$.

In this article, we show that the experimentally observed Raman 2D line width is a measure of nanometre-scale strain variations in graphene on insulating substrates, that is, it contains valuable information on local (that is, nanometre-scale) flatness, lattice deformations and crystal quality of graphene. Our findings solve the long-standing question of the nature of the observed broadening of the Raman 2D line and also link this quantity to the electronic transport properties of graphene, making it a valuable quantity for classifying the quality of graphene. To prove that the experimentally observed $2 \mathrm{D}$ line width depends on sub- spot-size strain variations and lattice deformations, we employ the following strategy.

We start by showing that by combining Raman spectroscopy with magnetic fields, electronic broadening contributions for the Raman G line width can be strongly suppressed. Since in perpendicular magnetic fields the electronic states in graphene condense into Landau levels (LLs), the interaction between electronic excitations and lattice vibrations becomes $B$-field dependent. In agreement with existing theory ${ }^{19-22}$ and experiments $^{23,24}$, we demonstrate that by applying a perpendicular $B$-field of $\sim 8 \mathrm{~T}$, the $\mathrm{G}$ line becomes almost independent of electronic properties such as charge carrier doping, screening, or electronic broadening.

We observe that, under these conditions, the $G$ line width nevertheless exhibits strong variations across graphene flakes. In particular, we show that the G line width is significantly increased in regions where the graphene flake features bubbles and folds, that is, in correspondence with increased structural deformations.

Finally, we show that at $8 \mathrm{~T}$, there is a (nearly) linear dependence between the G line width and the $2 \mathrm{D}$ line width, implying that there is a common source of line broadening. According to the previous points, the broadening must be related to structural lattice deformations. This finding is further supported by a detailed analysis of the relation between the area of the 2D peak and its line width. By analysing the relation between the G and 2D line width, we find that nanometre-scale strain variations constitute a dominant contribution to the observed line broadenings. Importantly, the $2 \mathrm{D}$ line has been shown to only very weakly depend on the $B$-field ${ }^{25}$, implying that no magnetic field is required to extract information on nanometre-scale strain variations from the $2 \mathrm{D}$ line width, which makes this quantity interesting for practical applications.

\section{Results}

Sample characterization. The investigated graphene (Gr) sheet is partly encapsulated in hexagonal boron nitride $(\mathrm{hBN})$ and partly
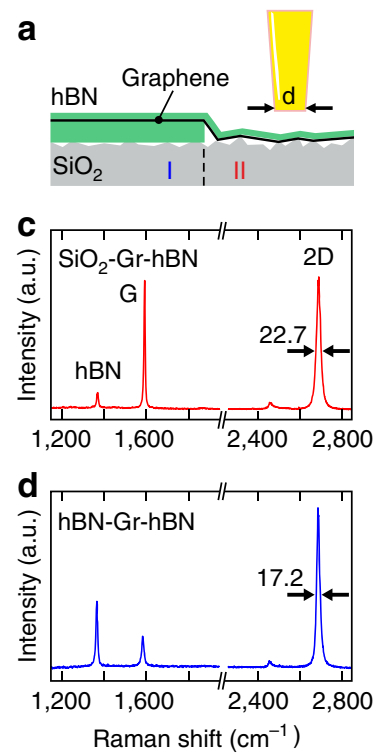
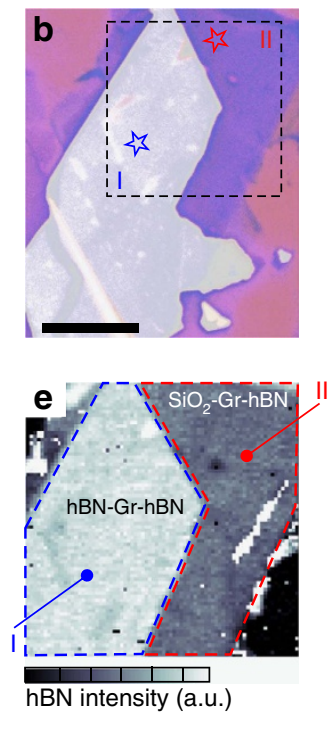
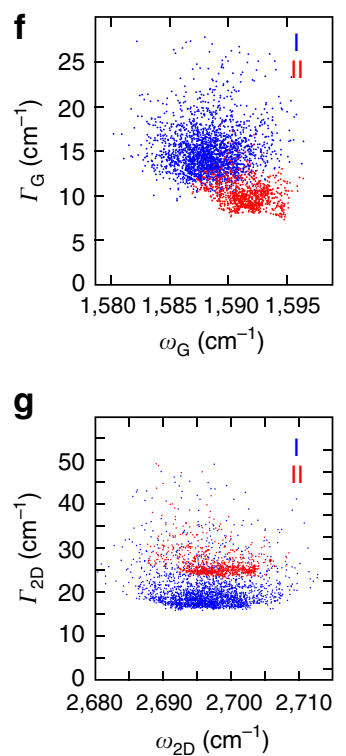
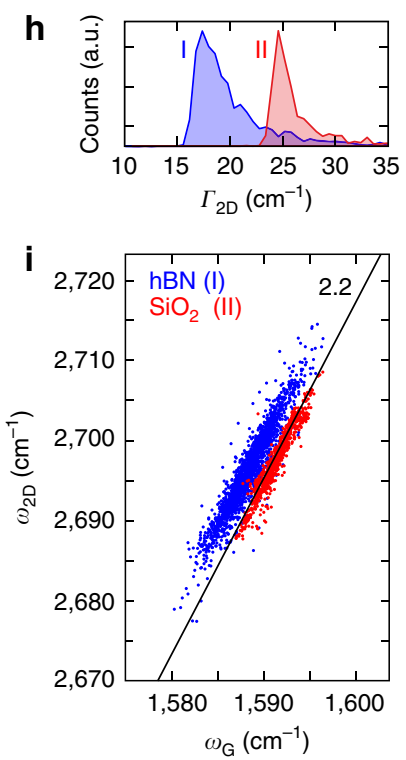

Figure 1 | Graphene sample characterization. (a) Schematic representation of cross-section of the investigated sample highlighting the different regions I (hBN-Gr-hBN) and II ( $\mathrm{SiO}_{2}-\mathrm{Gr}-\mathrm{hBN}$ ). (b) Optical image of a Gr-hBN heterostructure resting partly on hBN and $\mathrm{SiO}_{2}$. Scale bar, $10 \mu \mathrm{m}$. (c,d) Raman spectrum taken on the $\mathrm{SiO}_{2}-\mathrm{Gr}-\mathrm{hBN}$ (c) and hBN-Gr-hBN (d) areas. The positions where the spectra were taken are marked by a blue and a red star, respectively (b). (e) Raman map of the intensity of the $\mathrm{hBN}$ peak. The dashed lines mark the regions I and II. (f) $\Gamma_{\mathrm{G}}$ versus $\omega_{\mathrm{G}}$ recorded on various spots on regions I (blue) and II (red) of the sample. (g) $\Gamma_{2 D}$ versus $\omega_{2 D}$ recorded on various spots on regions I (blue) and II (red) of the sample. (h) Histograms of $\Gamma_{2 \mathrm{D}}$ recorded on various spots on regions I (blue) and II (red) of the sample. (i) $\omega_{2 \mathrm{D}}$ versus $\omega_{\mathrm{G}}$ recorded on various spots on regions I (blue) and II (red) of the sample. 
sandwiched between $\mathrm{SiO}_{2}$ and hBN as illustrated in Fig. 1a. An optical image of our sample is shown in Fig. 1b. In contrast to graphene encapsulated in $\mathrm{hBN}$, graphene flakes supported by $\mathrm{SiO}_{2}$ usually feature lower carrier mobilities of around $10^{3}-10^{4} \mathrm{~cm}^{2} /(\mathrm{Vs})$, indicating a detrimental influence of $\mathrm{SiO}_{2}$ on the electronic properties of graphene. In this regard, our structure gives us the invaluable capability of probing a single graphene sheet exposed to two different substrates (regions I and II in Fig. 1a,b). The sample is fabricated with a dry and resist-free transfer process following Wang et al. ${ }^{26}$ and Engels et al. ${ }^{27}$, where we pick up an exfoliated graphene flake with an hBN flake and deposit it onto the $\mathrm{hBN}-\mathrm{SiO}_{2}$ transition area of the substrate. A typical Raman spectrum of graphene supported by $\mathrm{SiO}_{2}$ and covered by $\mathrm{hBN}$, taken at the position of the red star in Fig. 1b, is shown in Fig. 1c. The characteristic hBN line as well as the graphene $\mathrm{G}$ and $2 \mathrm{D}$ lines can be clearly identified. At first glance, the spectra recorded in the hBN-Gr-hBN area look similar (see Fig. 1d, taken at the position marked by the blue star in Fig. 1b). However, it is evident that the full-width at half-maximum (FWHM) of the $2 \mathrm{D}$ line, $\Gamma_{2 \mathrm{D}}$, is significantly smaller.

The confocal nature of our Raman setup enables us to do spatially resolved measurements. An example of a Raman map is shown in Fig. 1e, where the spatially resolved intensity of the hBN line is depicted. The $h B N$ and $\mathrm{SiO}_{2}$ areas can be clearly distinguished in the map (see highlighted regions I and II). While analysing the Raman spectra of every point on the map, it is evident that the G lines recorded in the hBN-encapsulated area are broader than in the $\mathrm{SiO}_{2}$ supported area (compare red and blue data points in Fig. 1f). This is a clear indication of reduced charge carrier doping induced by the hBN substrate compared with $\mathrm{SiO}_{2}$. In fact, at low charge carrier doping, the phonon mode can decay into electron-hole pairs, which results in a broadening of the G peak ${ }^{5,28}$. For the $2 \mathrm{D}$ line, in contrast, the $\Gamma_{2 \mathrm{D}}$ recorded in the hBN-encapsulated area is mostly between $17 \mathrm{~cm}^{-1}$ and $20 \mathrm{~cm}^{-1}$, while it is above $22 \mathrm{~cm}^{-1}$ in the $\mathrm{SiO}_{2}$ area (see blue and red curves in the histogram of Fig. 1h, respectively). Note that both $\Gamma_{2 \mathrm{D}}$ and $\Gamma_{\mathrm{G}}$ do not depend on the respective frequencies $\omega_{2 \mathrm{D}}$ and $\omega_{\mathrm{G}}$ (Fig. 1f,g). In Fig. 1i the position of the $\mathrm{G}$ and 2D lines for every spectrum obtained on the investigated graphene sheet are displayed. For both substrates, the data points scatter along a line with a slope of 2.2. This slope coincides with the ratio of strain-induced shifts (that is, of the related Grüneisen parameters) of the Raman $\mathrm{G}$ and $2 \mathrm{D}$ modes ${ }^{29}$. This indicates that there are significant strain variations on both substrates across the entire graphene layer. Assuming the strain to be of biaxial nature, the spread of the data points translates into a maximum, micrometre-scale strain variation of $\sim 0.14 \%$ (ref. 29). The offset of the $\mathrm{SiO}_{2}$ and hBN data points can be understood in terms of the higher charge carrier doping induced by the $\mathrm{SiO}_{2}$ substrate, which shifts the data points towards higher values of $\omega_{\mathrm{G}}$ (ref. 5), and differences in the dielectric screening of $\mathrm{hBN}$ and $\mathrm{SiO}_{2}$ that effectively shift the $2 \mathrm{D}$ line position ${ }^{30}$. Since the data stems from a single graphene flake that has undergone identical fabrication steps for both substrate regions, the difference in charge carrier doping is unambiguously because of the two different substrate materials.

Suppressing electronic broadening with a magnetic field. For a more refined comparison of the Raman spectra on both substrates, we seek to suppress the effects on the G line arising from these differences in charge carrier doping. We therefore minimize the influence of the electronic system on the Raman G line by applying a perpendicular magnetic field. In the presence of a perpendicular magnetic field, the electronic states in graphene condense into LLs. The coupling of these LLs to the G mode is well understood ${ }^{19,20}$ and experimentally confirmed ${ }^{22-24,31-36}$. When a LL transition energetically matches the G mode phonon, the position of the G line is shifted and its line width increases. An example for the evolution of the Raman $G$ peak with magnetic field, taken on the hBN sandwich area, is shown in Fig. 2a. The individual spectra are offset for clarity. For a detailed analysis, single Lorentzians are fitted to every spectrum. The resulting frequency, $\omega_{\mathrm{G}}$, and FWHM, $\Gamma_{\mathrm{G}}$, are displayed in Fig. $2 \mathrm{~b}, \mathrm{c}$, respectively. The arrow at $B=3.7 \mathrm{~T}$ (Fig. $2 \mathrm{c}$ ) shows a value of the magnetic field where a LL transition is energetically matched with the phonon, leading to a broadening of the $\mathrm{G}$ line. However, at magnetic fields around $8 \mathrm{~T}$, no LL transition is energetically close to the G mode, as illustrated in Fig. 2d, where the energies of the relevant LL transitions as a function of magnetic field are compared with the energy of the G mode phonon. Consequently, at this high magnetic field the influence of the electronic system on the position and width of the G line is minimized. Note that this effect is independent of the charge carrier density and the exact values of the broadening of the LL transitions assuming that the latter are within a reasonable range as found by other studies $^{24,35}$. Thus, the residual broadening of the $\mathrm{G}$ line is most likely determined by phonon-phonon scattering and averaging effects over different strain values that vary on a nanometre scale.

Strain variations within the laser spot. To demonstrate that this applies to the entire sample, we first show that the broadening of the electronic states is low enough on the entire hBN-Gr-hBN area. In Fig. 3a,b, we show maps of $\Gamma_{\mathrm{G}}$ at $B=0$ and $3.8 \mathrm{~T}$, respectively. On the hBN part, the width of the G line shows the
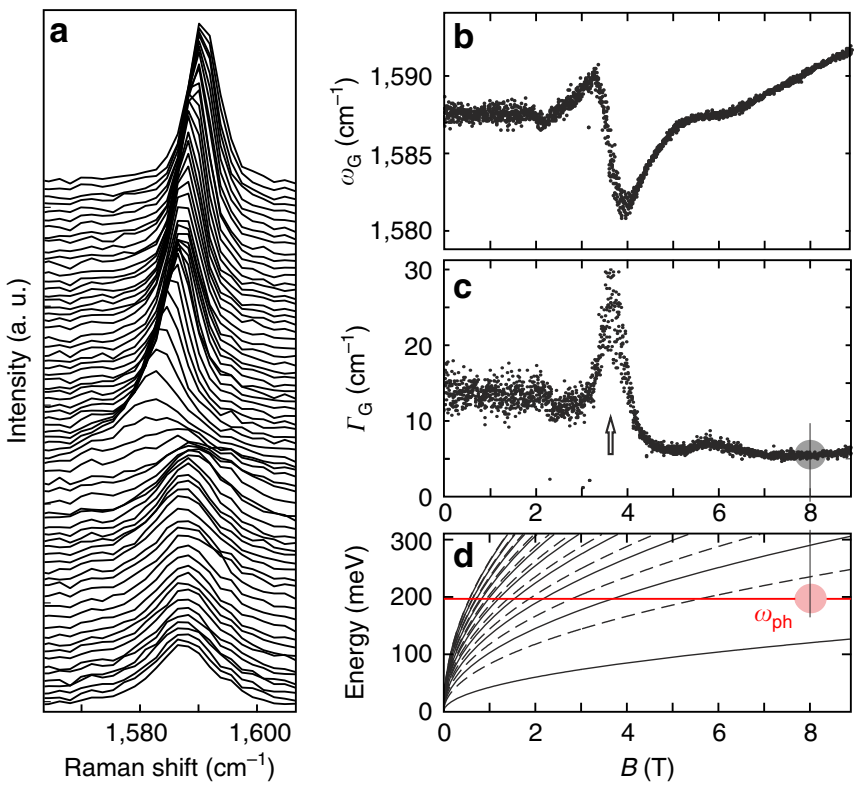

Figure 2 | Magneto-Raman spectroscopy. (a) Raman spectra recorded as a function of magnetic field, ranging from 0 T (bottom spectrum) to $8.9 \mathrm{~T}$ (top spectrum). The spectra are vertically offset for clarity. (b,c) Frequency, $\omega_{\mathrm{G}}$, and $\mathrm{FWHM}, \Gamma_{\mathrm{G}}$ of the $\mathrm{G}$ peak as a function of magnetic field as obtained from Lorentzian fits to the data shown in $\mathbf{a}$. The arrow (c)

highlights a value of the magnetic field at which the phonon is energetically matched to a LL transition. (d) Evolution of the energies of LL transitions with magnetic field. The full lines represent inter-band transitions in which the LL index changes by one. The dashed lines represent inter-band transitions in which the $L L$ index does not change. The red line represents the $\mathrm{G}$ mode phonon frequency at zero $B$ field. The circled region in $(\mathbf{c}, \mathbf{d})$ highlights the region in which no LL transitions energetically match the $\mathrm{G}$ mode phonon. 
a

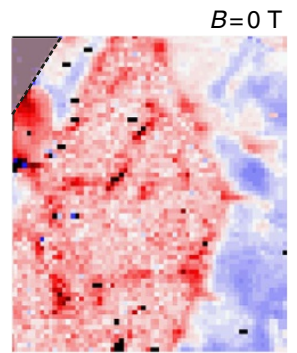

d

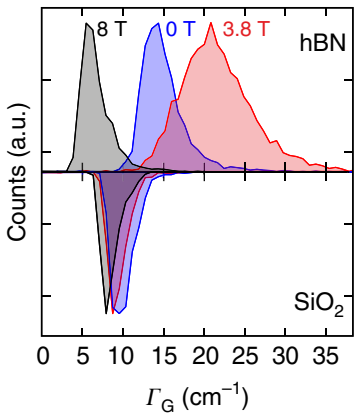

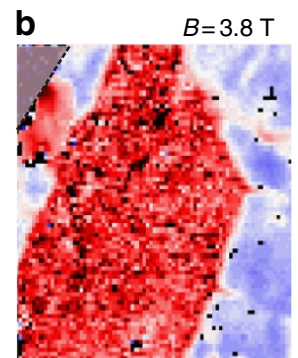

e

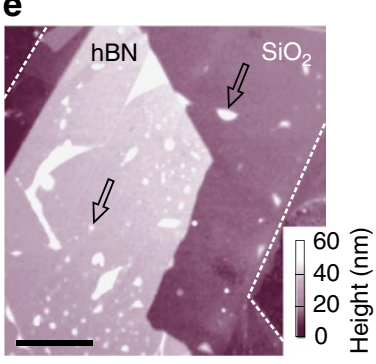

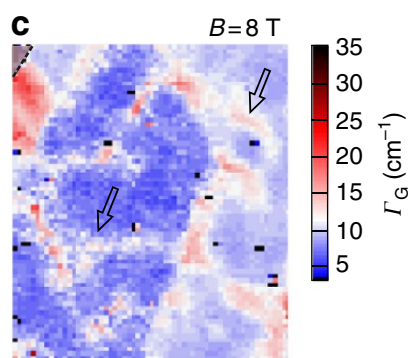

f

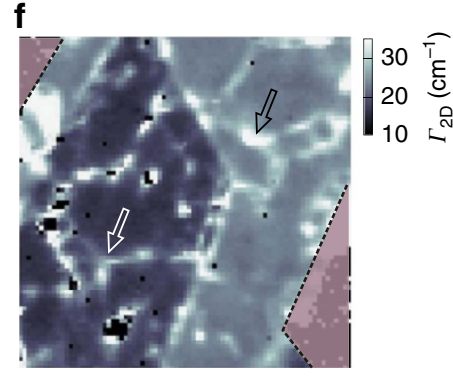

Figure 3 | Sample morphology probed by Raman spectroscopy. (a-c) Raman maps of the FWHM of the $G$ peak, $\Gamma_{G}$, taken at different magnetic fields, $B=0 \mathrm{~T}(\mathbf{a}), 3.8 \mathrm{~T}$ (b) and $8 \mathrm{~T}$ (c). The different regions I and II (labelled in Fig. 1a) can be well distinguished in all three panels. (d) Histograms of $\Gamma_{\mathrm{G}}$ for the different magnetic fields, $B=0 \mathrm{~T}$ (blue), 3.8 T (red), $B=8 \mathrm{~T}$ (grey) and the two substrates hBN (top panel) and $\mathrm{SiO}_{2}$ (bottom panel). (e) SFM image of the investigated sample. The scale bar represents $5 \mu \mathrm{m}$. (f) Raman map of $\Gamma_{2 \mathrm{D}}$ recorded at $0 \mathrm{~T}$. The arrows highlight mechanical folds visible in the SFM image as well as in the Raman maps (c,e,f). SFM, scanning force microscopy.

resonant behaviour depicted in Fig. 2c (see also histogram in Fig. 3d). This effect happens throughout the entire hBN area, independent of the local doping and strain values and independent of possible local folds and bubbles. The suppression of magneto-phonon resonances on the $\mathrm{SiO}_{2}$ substrate can be attributed to the higher charge carrier density. At higher charge carrier density the needed LL transitions are blocked by the Pauli principle. In the next step, we tune the magnetic field to $8 \mathrm{~T}$, where the electronic influences on the Raman $G$ line are at a minimum. A map of $\Gamma_{\mathrm{G}}$ over the entire flake at a magnetic field of $8 \mathrm{~T}$ is shown in Fig. $3 \mathrm{c}$. Distinct features across the whole sample are visible as regions with increased line width. A comparison with a scanning force microscope image of the sample (Fig. 3e) reveals that many of these regions can be associated with folds and bubbles most likely induced during the fabrication process, some of which even cross the border between the underlying hBN and $\mathrm{SiO}_{2}$ substrate regions.

As electronic broadening effects are suppressed at $8 \mathrm{~T}$, the increased line width of the $G$ line in the vicinity of these lattice deformations arises from enhanced phonon-phonon scattering and/or an averaging effect over varying nanometre-scale strain conditions.

Interestingly, the same features can also be identified in a $\Gamma_{2 \mathrm{D}}$ map recorded at $B=0 \mathrm{~T}$, shown in Fig. 3f. This strongly suggests that the lattice deformations identified at $8 \mathrm{~T}$ in $\Gamma_{\mathrm{G}}$ also cause a broadening of the $2 \mathrm{D}$ mode. The same trend is highlighted in Fig. $4 \mathrm{a}$, where we show the relation of $\Gamma_{\mathrm{G}}$ and $\Gamma_{2 \mathrm{D}}$ for all recorded Raman spectra at $8 \mathrm{~T}$. The additional teal data points stem from a $\mathrm{Gr}-\mathrm{SiO}_{2}$ sample and the orange star originates from a different hBN-Gr-hBN sandwich structure with all data having been obtained at $8 \mathrm{~T}$. Notably, the points from all substrate regions lie on one common line. From this linear relation between $\Gamma_{2 \mathrm{D}}$ and $\Gamma_{\mathrm{G}}$ (Fig. 4a), we conclude that there must be a common source of line broadening, which is connected to structural deformations. This is mainly due to the fact that at $8 \mathrm{~T}$ the G-line broadening is only very weakly affected by electronic contributions (see above). The range of the presented scatter plot can be extended by including data recorded on low-quality graphene samples with significant doping, as shown in Fig. 4b. Here, no magnetic field but high doping (corresponding to Fermi energies much higher than half of the phonon energy $\hbar \omega_{\mathrm{ph}} / 2 \approx 100 \mathrm{meV}$ ) is used to suppress Landau damping of the $G$ mode, leaving $\Gamma_{\mathrm{G}}$ unaffected from electronic contributions. The coloured data points stem from Raman maps $(B=0 \mathrm{~T})$ of chemical vapour deposition (CVD)-grown graphene flakes that were transferred onto $\mathrm{SiO}_{2}$ by a wet chemistry-based transfer. These graphene sheets contain doping values of $n_{\mathrm{el}}>3 \times 10^{12} \mathrm{~cm}^{-2}$, which corresponds to Fermi energies $E_{\mathrm{F}}>200 \mathrm{meV}$ (Supplementary Figs 1 and 2). The data points show the same trend as the values obtained at $8 \mathrm{~T}$ (grey data points in Fig. 4b) and even extend the total range of the dependence to higher values of $\Gamma_{2 \mathrm{D}}$.

\section{Discussion}

Although the linear relation between $\Gamma_{\mathrm{G}}$ and $\Gamma_{2 \mathrm{D}}$ in Fig. $4 \mathrm{a}, \mathrm{b}$ shows that structural deformations also broaden the $2 \mathrm{D}$ line, it is less straightforward to identify the actual mechanism of broadening. In principle, it is possible that the high values of $\Gamma_{2 \mathrm{D}}$ around folds and bubbles are due to a combination of increased phonon-phonon scattering, averaging effects over different strain values within the laser spot and reduced electronic life times. However, interestingly the slopes in Fig. 4a,b are around 2.2 (see black lines). This is a remarkable resemblance to the straininduced frequency shifts of both modes (compare Fig. 1i). This provides very strong indication that averaging over different strain values, which vary on a nanometre scale (see Fig. $4 \mathrm{c}$ ), play an important role in the broadening of the experimentally observed 2D line. This averaging effect broadens the $\mathrm{G}$ and $2 \mathrm{D}$ line by the same ratio as their peak positions shift for fixed average strain values explaining the slope of 2.2 between $\Gamma_{\mathrm{G}}$ and 

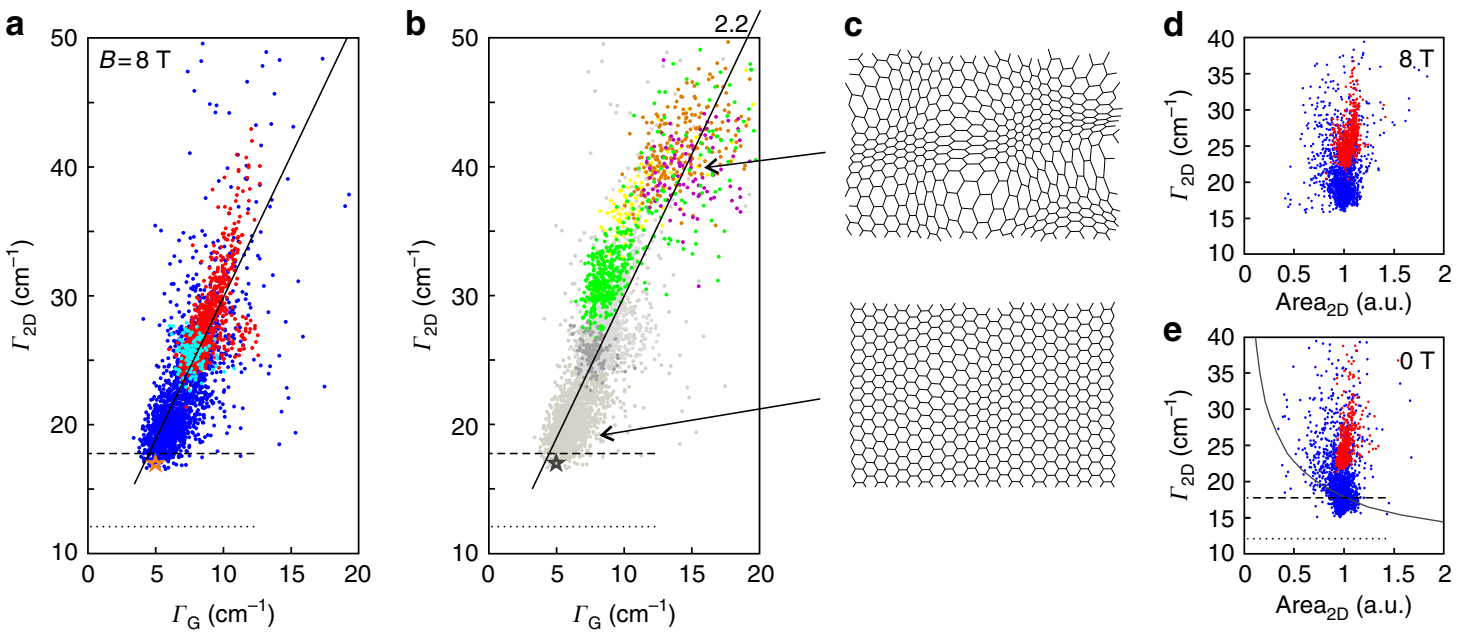

Figure 4 | Nanometre-scale strain variations. (a) $\Gamma_{G}$ versus $\Gamma_{2 D}$ recorded on various points on the $h B N$ part (blue) and $\mathrm{SiO}_{2}$ (red) of the sample at a magnetic field of $8 \mathrm{~T}$. Additional data points from a graphene-on- $\mathrm{SiO}_{2}$ sample (teal) and a second $\mathrm{hBN}-\mathrm{Gr}-\mathrm{hBN}$ sample (orange star) are shown. (b) The data points of (a) are depicted in grey. The coloured data are recorded on four different CVD graphene flakes on $\mathrm{SiO}_{2}$ substrate at 0 T. All four samples have doping values of $n_{\mathrm{el}}>3 \times 10^{12} \mathrm{~cm}^{-2}$, such that Landau damping of the $\mathrm{G}$ line is suppressed. The dashed and dotted lines in (a,b) indicate the calculated values of $\Gamma_{2 \mathrm{D}}$ from DFT calculations including electron-phonon and phonon-phonon broadening (dotted line) and electron-phonon, electroneletron and phonon-phonon broadening (dashed line). (c) Two schematic illustrations of nanometre-scale strain variations (top: large variations; bottom: small variations). (d) $\Gamma_{2 \mathrm{D}}$ versus the integrated area of the $2 \mathrm{D}$ peak as obtained from single Lorentzian fits for the $\mathrm{hBN}$ part (blue) and $\mathrm{SiO}_{2}(\mathrm{red})$ measured at $8 \mathrm{~T}$. Both data clouds are scaled to an average area $_{2 \mathrm{D}}$ value of one. (e) Similar plot as in panel (d) but for $0 \mathrm{~T}$. The solid black line is the calculated dependence of $\Gamma_{2 D}$ and area $_{2 D}$ for varying electronic broadening from the first-principles calculations, specified in the text and in Venezuela et al. ${ }^{38}$. The dashed and dotted black lines are the same as in $(\mathbf{a}, \mathbf{b})$. DFT, density-functional theory.

$\Gamma_{2 \mathrm{D}}$ (Supplementary Fig. 3), which we demonstrate with a simple toy model as shown in Fig. 5. Each individual Raman process that takes place within the laser spot is subject to a different amount of strain since the latter varies across the laser spot. Each of the corresponding Raman peaks is thus shifted by a different amount (see blue and dashed cyan curves in Fig. 5a). The intrinsic broadening of each individual Raman process is assumed to be $\Gamma_{\mathrm{G}}=5 \mathrm{~cm}^{-1}$ and $\Gamma_{2 \mathrm{D}}=17 \mathrm{~cm}^{-1}$. Due to the size of the laser spot, the sum of several of these individual Raman processes is recorded, with the resulting peak being given by the sum of the individual peaks (see blue curve in Fig. 5b). Following the data analysis of our measurements, the resulting curve is fitted by a single Lorentzian (red curve in Fig. 5b). To simulate the effect of this statistical broadening mechanism on the width of the resulting Raman peak, we simulate statistical strain distributions for several laser spots that are subject to different amounts of strain variation $\Delta \varepsilon$ in Fig. $5 c$. For each of the red points, 20 strain values were randomly generated. Each set of strain values follows a Gaussian distribution centred at $\bar{\varepsilon}=-0.1 \%$ with a width varying from $\Delta \varepsilon=0$ to $0.15 \%$. The dashed black line has a slope of 2.2 and matches the distribution of the red points, illustrating that averaging over nanometre-scale variations leads to a linear dependence of $\Gamma_{\mathrm{G}}$ and $\Gamma_{2 \mathrm{D}}$.

We are aware that the low charge carrier densities in the $\mathrm{hBN}$-encapsulated area might result in a narrowing of the $2 \mathrm{D}$ mode by three to four wave numbers ${ }^{37}$. However, the large differences of $\Gamma_{2 \mathrm{D}}$ on the order of $20-30 \mathrm{~cm}^{-1}$ on both substrates cannot be explained by the differences in charge carrier doping $7,37,38$.

Interestingly, the lowest $\Gamma_{2 \mathrm{D}}$ observed in our experiments are very close to the value that we compute from first-principles as by Venezuela et al. ${ }^{38}$ assuming an undoped, defect-free and stress-free sample of graphene (horizontal dashed and dotted lines in Fig. 4a,b). In such an approach, the width of the 2D peak is determined by the anharmonic decay rate of the two phonons involved $\left(5.3 \mathrm{~cm}^{-1}\right.$ according to Paulatto et al. $\left.{ }^{39}\right)$, and, indirectly,
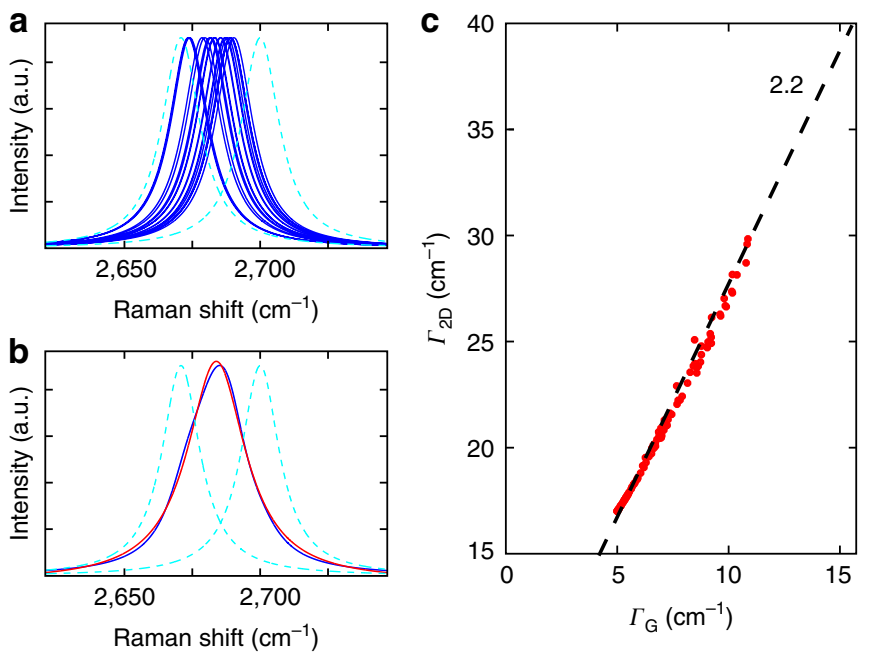

Figure 5 | Illustration of line broadening due to averaging effects.

$(\mathbf{a}, \mathbf{b})$ Individual Raman processes within the laser spot (a) add up to a broad Raman peak that is recorded (blue peak in $\mathbf{b}$ ). The red curve in (b) is a Lorentzian fit to the blue line. The dashed cyan lines, representing the two outmost individual Raman processes, are the same in $(\mathbf{a}, \mathbf{b})$ and serve as a guide to the eye. (c) Width of the statistical broadened Raman $G$ and 2D peaks as obtained from a simple statistical model. The procedure described in $(\mathbf{a}, \mathbf{b})$ was performed for different sets of strain variations. Each set of strain values follows a Gaussian distribution centred at $\bar{\varepsilon}=-0.1 \%$ with a width varying from $\Delta \varepsilon=0-0.15 \%$. The resulting $\mathrm{G}$ and $2 \mathrm{D}$ peaks are fitted with a single Lorentzian with the respective widths represented by a red point. The dashed black line has the slope of 2.2. It matches to the generated data points indicating that strain variations within the laser spot broaden the $\mathrm{G}$ and $2 \mathrm{D}$ lines by this ratio. 
by the broadening of the electron and hole, denoted as $\gamma$ in Venezuela et al. ${ }^{38}$ (see also Basko ${ }^{40}$ ). According to Reference Venezuela et al. $^{38}$, the electron-phonon contribution to $\gamma$ is $81.9 \mathrm{meV}$ for electronic states in resonance with the $2.33 \mathrm{eV}$ laserlight. With such a value of $\gamma$ we obtain a $\Gamma_{2 \mathrm{D}}$ of $12.1 \mathrm{~cm}^{-1}$ (dotted lines in Fig. 4a,b,e). If, following Herziger et $a l^{41}$, we double the value of $\gamma$ to account for the electron-electron scattering, we obtain a $\Gamma_{2 \mathrm{D}}$ of $17.9 \mathrm{~cm}^{-1}$ (dashed lines in Fig. 4a,b,e), in close agreement with the lowest measured values. In principle, the observed increase of $\Gamma_{2 \mathrm{D}}$ with respect to its minimum value could be attributed to an increase of the electronic broadening $\gamma$, due to doping (increasing the electron-electron scattering) or to the presence of defects (increasing the electron-defect scattering) ${ }^{38,40,42}$. By investigating the relation between $\Gamma_{2 \mathrm{D}}$ and the integrated area of the $2 \mathrm{D}$ peak $\left(\operatorname{area}_{2 \mathrm{D}}\right)$ we can exclude such a hypothesis. In Fig. $4 \mathrm{~d}$,e we show scatter plots of $\Gamma_{2 \mathrm{D}}$ versus the region-normalized area $_{2 \mathrm{D}}$ for both $B=8 \mathrm{~T}$ and $B=0 \mathrm{~T}$, highlighting the very weak $B$-field dependence of $\Gamma_{2 \mathrm{D}}$. More importantly, we observe that the area of the $2 \mathrm{D}$ peak does not depend on $\Gamma_{2 \mathrm{D}}$, contrary to what is expected in presence of a variation of the electronic broadening $\gamma$ (refs 38,40,42). In particular the measured data does not follow the calculated dependence of $\Gamma_{2 \mathrm{D}}$ on area $_{2 \mathrm{D}}$, reported in Fig. $4 \mathrm{e}$, obtained in the calculation by varying electronic broadening $\gamma$. This dismisses differences in the electronic broadening as a main mechanism for the observed variations of $\Gamma_{2 D}$.

Finally, our finding that the 2D line depends on nanometrescale strain inhomogeneities is also in good agreement with highresolution scanning tunnelling microscopy measurements, which reveal that graphene on $\mathrm{SiO}_{2}$ forms short-ranged corrugations, while graphene on hBN features significantly flatter areas ${ }^{43}$.

In summary, we showed that by using a magnetic field of $8 \mathrm{~T}$ to strongly suppress the influence of the electronic contributions on the Raman G line width, the latter can be used as a measure for the amount of nanometre-scale strain variations. Most importantly, we observed a nearly linear dependence between the $\mathrm{G}$ and $2 \mathrm{D}$ line widths at $8 \mathrm{~T}$ independent of the substrate material, indicating that the dominating source of the spread of the broadening of both peaks is the same. From the slope $\Delta \Gamma_{2 \mathrm{D}} / \Delta \Gamma_{\mathrm{G}}$ of around 2.2 , we deduce that averaging effects over nanometre-scale strain variations make a major contribution to this trend. Since the $2 \mathrm{D}$ line width shows only a very weak dependence on the $B$ field, this quantity can even be used without a magnetic field to gain information on the local strain homogeneity and thus on the structural quality of graphene. These insights can be potentially very valuable for monitoring graphene fabrication and growth processes in research and industrial applications, where a fast and non-invasive control of graphene lattice deformations is of great interest.

\begin{abstract}
Methods
Raman spectroscopy measurements. The room temperature Raman spectra were acquired using a commercial Witec system with a laser excitation of $532 \mathrm{~nm}$ $(2.33 \mathrm{eV})$ delivered through a single-mode optical fibre, where the spot size is limited by diffraction. Using a long working distance focusing lens with a numerical aperture of 0.80 , we obtained a spot size of $\sim 400-500 \mathrm{~nm}$. For the lowtemperature Raman measurements, we employ a commercially available confocal Raman setup that allows us to perform spatially resolved experiments at a temperature of $4.2 \mathrm{~K}$ and magnetic fields of up to $9 \mathrm{~T}$. We use an excitation laser wavelength of $532 \mathrm{~nm}$ with a spot diameter on the sample of $\sim 500 \mathrm{~nm}$. For detection, we use a single-mode optical fibre and a charge-coupled spectrometer with a grating of 1,200 lines $\mathrm{mm}^{-1}$. All measurements are performed with linear laser polarization and $\mathrm{a} \times 100$ objective.
\end{abstract}

First-principles calculations. For the computation of the double-resonant Raman cross-section we employ an approach based on Fermi's golden rule generalized to the fourth perturbative order as described in detail in Reference Venezuela et al. ${ }^{38}$.
In this approach, electron-light, and electron-phonon scattering matrix elements are explicitly calculated and the phonon and electronic dispersions reproduce calculations based on density-functional theory corrected with GW. Converged results are obtained using $480 \times 480$ and $240 \times 240$ grids in the Brillouin zone for the electron and phonon wave-vectors, respectively. The finite phonon life time is taken into account by broadening the Raman intensity with $5.3 \mathrm{~cm}^{-1}$ wide Lorentzians ${ }^{39}$. We varied the electron broadening ( $\gamma$ in eq. (5) from Venezuela et al. ${ }^{38}$ ) from 16.4 to $344 \mathrm{meV}$ and then we determined $\Gamma_{2 \mathrm{D}}$ as a function of area $_{2 \mathrm{D}}$ For a laser energy of $2.33 \mathrm{eV}$, the electron-phonon contribution for the electronic broadening is $81.9 \mathrm{meV}$, which leads to $\Gamma_{2 \mathrm{D}}=12.1 \mathrm{~cm}^{-1}$. However, when we choose $\gamma$ to be twice this value, to account for additional electron-electron interaction ${ }^{41}$, we obtain $\Gamma_{2 \mathrm{D}}$ equal to $17.9 \mathrm{~cm}^{-1}$. These values can be understood as a theoretical expectation of the $2 \mathrm{D}$ line width for a perfect graphene lattice disregarding any broadening from averaging effects over different strain values within the laser spot.

\section{References}

1. Novoselov, K. S. et al. A roadmap for graphene. Nature 490, 192-200 (2012)

2. Ferrari, A. et al. Raman spectrum of graphene and graphene layers. Phys. Rev. Lett. 97, 187401 (2006).

3. Graf, D. et al. Spatially resolved Raman spectroscopy of single- and few-layer graphene. Nano. Lett. 7, 238-242 (2007).

4. Ferrari, A. C. Raman spectroscopy of graphene and graphite: disorder, electronphonon coupling, doping and nonadiabatic effects. Solid State Commun. 143, 47-57 (2007).

5. Yan, J., Zhang, Y., Kim, P. \& Pinczuk, A. Electric field effect tuning of electronphonon coupling in graphene. Phys. Rev. Lett. 98, 166802 (2007).

6. Pisana, S. et al. Breakdown of the adiabatic Born-Oppenheimer approximation in graphene. Nat. Mater. 6, 198-201 (2007).

7. Stampfer, C. et al. Raman imaging of doping domains in graphene on $\mathrm{SiO}_{2}$. Appl. Phys. Lett. 91, 241907 (2007).

8. Mohr, M., Maultzsch, J. \& Thomsen, C. Splitting of the Raman 2D band of graphene subjected to strain. Phys. Rev. B 82, 201409 (2010).

9. Huang, M., Yan, H., Heinz, T. F. \& Hone, J. Probing strain-induced electronic structure change in graphene by Raman spectroscopy. Nano. Lett. 10, 4074-4079 (2010).

10. Calizo, I., Balandin, A., Bao, W., Miao, F. \& Lau, C. Temperature dependence of the Raman spectra of graphene and graphene multilayers. Nano. Lett. 7, 2645-2649 (2007).

11. Balandin, A. A. et al. Superior thermal conductivity of single-layer graphene. Nano Lett. 8, 902-907 (2008).

12. Drögeler, M. et al. Nanosecond spin lifetimes in single-and few-layer graphene-hBN heterostructures at room temperature. Nano Lett. 14, 6050-6055 (2014).

13. Casiraghi, C. et al. Raman spectroscopy of graphene edges. Nano Lett. 9 , 1433-1441 (2009).

14. Mohiuddin, T. et al. Uniaxial strain in graphene by Raman spectroscopy: G peak splitting, Grüneisen parameters, and sample orientation. Phys. Rev. B 79, 205433 (2009).

15. Zabel, J. et al. Raman spectroscopy of graphene and bilayer under biaxial strain bubbles and balloons. Nano Lett. 12, 617-621 (2012).

16. Yoon, D., Son, Y.-W. \& Cheong, H. Strain-dependent splitting of the doubleresonance Raman scattering band in graphene. Phys. Rev. Lett. 106, 155502 (2011).

17. Couto, N. J. et al. Random strain fluctuations as dominant disorder source for high-quality on-substrate graphene devices. Phys. Rev. X 4, 041019 (2014).

18. Banszerus, L. et al. Ultrahigh-mobility graphene devices from chemical vapor deposition on reusable copper. Sci. Adv. 1, e1500222 (2015).

19. Ando, T. Magnetic oscillation of optical phonon in graphene. J. Phys. Soc. Jpn 76, 024712 (2007).

20. Goerbig, M., Fuchs, J.-N., Kechedzhi, K. \& Fal'ko, V. I. Filling-factor-dependent magnetophonon resonance in graphene. Phys. Rev. Lett. 99, 087402 (2007).

21. Kashuba, O. \& Fal'ko, V. I. Role of electronic excitations in magneto-Raman spectra of graphene. New. J. Phys. 14, 105016 (2012).

22. Qiu, C. et al. Strong magnetophonon resonance induced triple G-mode splitting in graphene on graphite probed by micromagneto Raman spectroscopy. Phys. Rev. B 88, 165407 (2013).

23. Yan, J. et al. Observation of magnetophonon resonance of Dirac fermions in graphite. Phys. Rev. Lett. 105, 227401 (2010).

24. Neumann, C. et al. Low B field magneto-phonon resonances in single-layer and bilayer graphene. Nano. Lett. 15, 1547-1552 (2015).

25. Faugeras, C. et al. Effect of a magnetic field on the two-phonon Raman scattering in graphene. Phys. Rev. B 81, 155436 (2010).

26. Wang, L. et al. One-dimensional electrical contact to a two-dimensional material. Science 342, 614-617 (2013).

27. Engels, S. et al. Impact of thermal annealing on graphene devices encapsulated in hexagonal boron nitride. Phys. Status Solidi B 251, 2545-2550 (2014). 
28. Casiraghi, C., Pisana, S., Novoselov, K., Geim, A. \& Ferrari, A. Raman fingerprint of charged impurities in graphene. Appl. Phys. Lett. 91, 233108 (2007).

29. Lee, J. E., Ahn, G., Shim, J., Lee, Y. S. \& Ryu, S. Optical separation of mechanical strain from charge doping in graphene. Nat. Commun. 3, 1024 (2012).

30. Forster, F. et al. Dielectric screening of the Kohn anomaly of graphene on hexagonal boron nitride. Phys. Rev. B 88, 085419 (2013).

31. Faugeras, C. et al. Magneto-Raman scattering of graphene on graphite: electronic and phonon excitations. Phys. Rev. Lett. 107, 036807 (2011).

32. Faugeras, C. et al. Probing the band structure of quadri-layer graphene with magneto-phonon resonance. New J. Phys. 14, 095007 (2012).

33. Faugeras, C. et al. Tuning the electron-phonon coupling in multilayer graphene with magnetic fields. Phys. Rev. Lett. 103, 186803 (2009).

34. Kossacki, P. et al. Circular dichroism of magnetophonon resonance in doped graphene. Phys. Rev. B 86, 205431 (2012).

35. Kim, Y. et al. Measurement of filling-factor-dependent magnetophonon resonances in graphene using Raman spectroscopy. Phys. Rev. Lett. 110, 227402 (2013).

36. Leszczynski, P. et al. Electrical switch to the resonant magneto-phonon effect in graphene. Nano. Lett. 14, 1460-1466 (2014).

37. Berciaud, S. et al. Intrinsic line shape of the Raman 2D-mode in freestanding graphene monolayers. Nano. Lett. 13, 3517-3523 (2013).

38. Venezuela, P., Lazzeri, M. \& Mauri, F. Theory of double-resonant Raman spectra in graphene: intensity and line shape of defect-induced and twophonon bands. Phys. Rev. B 84, 035433 (2011).

39. Paulatto, L., Mauri, F. \& Lazzeri, M. Anharmonic properties from a generalized third-order ab initio approach: theory and applications to graphite and graphene. Phys. Rev. B 87, 214303 (2013).

40. Basko, D. M. Theory of resonant multiphonon Raman scattering in graphene. Phys. Rev. B 78, 125418 (2008).

41. Herziger, F. et al. Two-dimensional analysis of the double-resonant 2D Raman mode in bilayer graphene. Phys. Rev. Lett. 113, 187401 (2014).

42. Basko, D., Piscanec, S. \& Ferrari, A. Electron-electron interactions and doping dependence of the two-phonon Raman intensity in graphene. Phys. Rev. B 80, 165413 (2009).

43. Lu, C.-P., Li, G., Watanabe, K., Taniguchi, T. \& Andrei, E. Y. $\mathrm{MoS}_{2}$ : choice substrate for accessing and tuning the electronic properties of graphene. Phys. Rev. Lett. 113, 156804 (2014).

\section{Acknowledgements}

We thank T. Khodkov for support during the measurements. Support by the Helmholtz Nanoelectronic Facility (HNF), the Deutsche Forschungsgemeinschaft through SPP 1459, the ERC (GA-Nr. 280140) and the EU project Graphene Flagship (contract no. NECT-ICT-604391), are gratefully acknowledged. P.V. acknowledges financial support from the Capes-Cofecub agreement. The work of S.V.R. was partially supported by NSF (ECCS-1509786) and the CORE grant from Lehigh University.

\section{Author contributions}

C.S., C.N., B.B. and S.V.R. conceived the experiment. C.N. and M.D. carried out the optical measurements. C.N. fabricated the exfoliated graphene samples. L.B. and M.S. fabricated the CVD graphene samples. K.W. and T.T. synthesized the hBN samples. P.V. and F.M. performed the first-principles calculations. C.N., S.R. and S.V.R. performed data analysis and theoretical analysis. C.N., S.R., S.V.R., B.B., P.V., F.M and C.S. co-wrote the manuscript. All authors discussed the results and commented on the paper.

\section{Additional information}

Supplementary Information accompanies this paper at http://www.nature.com/ naturecommunications

Competing financial interests: The authors declare no competing financial interests.

Reprints and permission information is available online at http://npg.nature.com/ reprintsandpermissions/

How to cite this article: Neumann, C. et al. Raman spectroscopy as probe of nanometrescale strain variations in graphene. Nat. Commun. 6:8429 doi: 10.1038/ncomms9429 (2015)

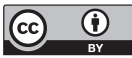

This work is licensed under a Creative Commons Attribution 4.0 International License. The images or other third party material in this article are included in the article's Creative Commons license, unless indicated otherwise in the credit line; if the material is not included under the Creative Commons license, users will need to obtain permission from the license holder to reproduce the material. To view a copy of this license, visit http://creativecommons.org/licenses/by/4.0/ 